\title{
Descripción de primeros registros micomórficos para el Ecuador
}

\author{
J. Paul Gamboa-Trujillo ${ }^{1,2,3,4,5,6^{*}}$, Miriam Cevallos ${ }^{7}$ \& Tatiana Gibertoni ${ }^{6}$
}

${ }^{1}$ Facultad de Ciencias Biológicas-Carrera de Biología Universidad Central del Ecuador-UCE, Ecuador. ${ }^{2}$ Laboratorio de Micología Aplicada, Facultad de Ingeniería Química-UCE, Ecuador. ${ }^{3}$ Laboratorio de Micología-Instituto de Biomedicina, Hospital Docente de Calderón CBM- Facultad de Ciencias Médicas-UCE, Ecuador. ${ }^{4}$ Sección Micológica del Herbario Alfredo Paredes QAP-UCE- Avenida América y Gato Sobral,

Ciudadela Universitaria, Ecuador. ${ }^{5}$ Sección Micológica del Herbario Nacional del Ecuador QCNE, Instituto Nacional de Biodiversidad INABIO, Rio Coca e Isla Bernardina, Quito, Pichincha, Ecuador. ' Universidade Federal de Pernambuco, Departamento de Micologia/CCB, Av. Prof. Nelson Chaves, s/nº, CEP: 50670-901, Recife, PE, Brazil. ${ }^{7}$ Museo Antropológico Antonio Santiana (UCE)

Email:.jpgamboa@uce.edu.ec / paulgamboativi@hotmail.com

\section{Resumen}

El Museo Arqueológico Antonio Santiana de la Universidad Central del Ecuador de la ciudad de Quito, posee un acervo de piezas arqueológicas importantes para el estudio del comportamiento social de los antepasados de esta región, constituyéndose en el sitio estratégico para localizar dos posibles figuras micomórficas. Una de ellas en diseño pictórico decorando un recipiente cóncavo con caracteres similares al gasteromicete Geastrum sp., perteneciente a la cultura Cuasmal; la segunda caracteriza un cáliz ceremonial de forma parecida a un macrohongo agaricomicete, realizada por la cultura Manteña. Estas piezas representan vestigios que explican el posible uso de macrohongos, vistos como organismos útiles y sagrados, utilizados para eventos rituales. También revelan la importancia de estudiar, mediante la bioarqueología, costumbres micófilas compartidas con otras comunidades del continente americano, con quienes existió un fuerte intercambio de productos y conocimientos que se expresan hasta nuestros días, aunque en menor grado por el advenimiento de la vida moderna y la mezcla de culturas.

Palabras clave: Micomórficos, macro hongos, micófilo, bioarqueología, Ecuador.

\section{Abstract}

The Archaeological Museum Antonio Santiana Central University of Ecuador, Quito; has a collection of archaeological importance to the study of social behavior of the ancestors of this region, becoming the strategic site to locate two possible micomorphical estructures. One of them in pictorial design decorating a concave bowl-like characters similar by Geastrum $\mathrm{sp}$. belonging to the Cuasmal culture. The second features is a ceremonial cup, similar to agaricales macrofungi by Man- 
teña culture. These pieces represent the possible traces that explain use of macrofungi, seen as beneficial and sacred rituals used for events. They also reveal the importance of studying, by bioarchaeology, mycophylous customs shared with other communities in America, with whom there was a heavy exchange of products and knowledge that are expressed to this day, to a lesser degree by the advent of modern life the mixture of cultures.

Keywords: Mycomorphic, macro fungi, mycophyllas, bioarchaeology, Ecuador.

\section{Introducción}

Pocos son los registros micomórficos que representan comportamientos micófilos de las culturas prehispánicas en América del Sur. No así, para las culturas ancestrales ubicadas en Mesoamérica los hongos macroscópicos eran, y aún son, considerados de gran importancia, principalmente para algunas culturas de la región centro-sureste de México y de la zona norte de Centroamérica, que utilizaban a estos organismos como medicamento, alimento y elemento mágico-ritual.

R.G. y V.P. Wasson, a quienes se los considera los padres de la etnomicología, describen a ídolos guatemaltecos en forma de hongo, descubiertos a principios del siglo XX por C. Sapper y S.de Borhéguy, quienes analizaron ídolos labrados en piedra, efectuados entre el siglo XIII AC y el XI DC (De Sahagún,1955)

Además, Schultes y Hofmann (1979) presentan una vasija con representaciones indígenas de una Amanita muscaria en el centro de la misma (Guzmán, 2001), siendo esta una de la evidencias del uso de este hongo por parte de los indígenas Nahuatl de Jalisco y Colima en México en el pasado. Estos indígenas fueran también considerados consumidores de varias especies alucinógenas (Guzmán, 2009), como lo estudiado para los Matlazincs y Nahuatls del centro de México, y Mazatecos, Chinentecs, Mixes, Zapotecos y Chatins en el Estado de Oaxaca. Este comportamiento micófilo fue probablemente compartido en el pasado con los indígenas del Nevado de Colima, Purepechas de Michoacan y Totonacs de Veracruz, quienes también usaban esos hongos (Guzmán, 2009). En consideración a todos los grupos de indígenas antes mencionados; Guzmán ( 2009) encontró en Schultes y Hofmann (1979) tres figuras indígenas de cerámica interesantes, que poseen una relación fuerte al uso tradicional de las especies alucinógenas de Psilocybe y sus efectos de gigantismo, mostrando cuatro indígenas danzando alrededor de un hongo alto Schultes y Hofmann,1979). Sin embargo, esta interpretación es equivocada, porque bajo el efecto de los hongos alucinógenos, es difícil permanecer de pie, caminar o danzar, por lo que los cuatro indígenas están realmente abrazándose para no caer y observando con admiración el hongo gigante ubicado en el centro (Guzmán, 2009); por estas características México es reconocido como un país con tradición ancestral en el uso de hongos en las diferentes categorías de consumo, denominados así como micófilos.

Dentro de estos hallazgos, también se registran un tipo de pectoral (Schultes y Bright, 1985) que demuestra las complejidades sobre el uso mágico-religioso, shamánico o ceremonial de plantas y hongos alucinógenos al sur de Pana- 
má, específicamente en la zona de Darién-Colombia, como pruebas del uso religioso en la Colombia prehispánica (Velandia et al. 2008).

En el Ecuador, fueron descritos algunos hongos comestibles como Lepiota callamba cuyo nombre vernacular es Kallamba, siendo este el único indicio de datos etnomicológicos en la antigüedad (Patouillard y Lagerheim, 1891), sin describir hallazgos micomorficos que prueben la importancia ancestral de estos organismos dentro de las culturas asentadas en este territorio antes de la llegada de los españoles.

De aquí que el presente trabajo tiene como objetivo principal analizar e identificar posibles figuras micomórficas depositadas y expuestas en dos de los museos arqueológicos importantes de la ciudad de Quito- Ecuador; la presencia de estas piezas arqueológicas demuestran comportamientos micófilos en el Ecuador, basados en la adoración y expresión de utilidad de estos organismos, considerados de vital importancia dentro del hábitat en donde coexistían.

\section{Materiales y Métodos}

Con el fin de incrementar información al estudio de la Etnomicología ecuatoriana, se realizaron visitas a museos ex situ e in situ ubicados en las diferentes ciudades del país, donde se exponen figuras antropológicas de las distintas culturas ancestrales del Ecuador. Fueran efectuadas observaciones a los siguientes museos: Museo Arqueológico Antonio Santiana de la Universidad Central del Ecuador (ex situ), ubicado en la ciudad de Quito, provincia de Pichincha; Museo Nacional
Banco del Central de Ecuador (ex situ) y Museo Antropológico de Agua Blanca (in situ), ubicados en la población de Agua Blanca, provincia de Manabí.

Cada una de las piezas fue analizada para la identificación de estructuras micomórficas de los diferentes macromicetos (Phylums Ascomycota y Basidiomycota), realizando un inventario preliminar etnomicológico y arqueológico que demuestren costumbres micófilas en las culturas ancestrales ecuatorianas.

\section{Resultados y Discusión}

De los tres sitios visitados, en uno de ellos (Museo Antonio Santiana) fueran encontradas dos figuras que presentan caracteres morfológicos semejantes a dos macromicetos (Agaricomycetes, Basidiomycota). La primera pertenece a una etnia pre-incaica que estuvo ubicada en el norte de la región Sierra del Ecuador (Figura 1) y una segunda descubierta en la región centro norte de la costa ecuatoriana (Figura 2).

Con los resultados, a continuación descritos, podemos considerar que el Ecuador es un país con costumbres micófilas, deduciendo la apreciación para los macromicetos por nuestros antepasados, dentro de varias categorías de uso tales como medicinales y principalmente rituales.

\section{Cultura Cuasmal}

Se registra una compotera de pie corto, encontrada en la zona central montañosa de los Andes ecuatorianos, en la provincia del Carchi, y que data de 1250-1532 dC. Esta pieza fue estudiada previamen- 
te por los investigadores Jacinto Jijón y Caamaño en 1917, Max Uhle en 1927 y Carlos Grijalba en 1937 (Grijalba, 1937; Porras, 1987).

El pueblo de los Pastos usaba la técnica de modelado en cerámica, decorada en negativo, blanco y rojo, y pulida con guijarro; diseñó en esta vasija ceremonial hemisférica figuras pictóricas posiblemente micomórficas. Estas figuras son parecidas a tres basidiomas con el exoperidio cerrado y lo que consideraríamos cuatro cuerpos fructíferos abiertos, mostrando estructuras parecidas a lacinias en su contorno y el endoperidio con el ostiolo al centro, asemejándose así a Geastrum sp. (Geastraceae, Geastrales) (Figura 1). Este hongo fue usado probablemente como remédio, puesto que según estudios realizados en la cuenca alta del Rio Intag, provincia de Imbabura, que colinda con la provincia del Carchi, las personas que habitan en este sector utilizan este hongo como cicatrizante, para curar cortes de piel ocasionados mientras recorren los senderos del bosque o trabajan en el campo. Para esto, presionan el endoperidio de Geastrum saccatum Fr., liberando las basidioesporas para luego colocarlas en la herida hasta que esta cicatrize. El nombre común como lo denominan a este interesante gasteromiceto es Sopapo o Pedo del Diablo (Gamboa-Trujillo, 2005). De aquí que, por el flujo de información de las etnias ancestrales, el conocimiento paso de generación en generación, prevaleciendo hasta la actualidad.

En algunas culturas prehispánicas mexicanas, se observan representaciones pictóricas y esculturas de forma convexa demostrando el margen del píleo vuelto hacia adentro, representando macrohongos cortados transversalmente (Wasson,
1980; Velandia et al., 2008) o, como en este caso, en forma de estrellas y circunferencias (Fig 1).

No existen vestigios directos sobre las costumbres ceremoniales de esta cultura, pero por la cantidad de decoración cerámica se puede intuir de un culto a la naturaleza debido a la presencia de danzantes pintados en las superficies de otros vasos y compoteras. Esos danzantes realizaban actos de adoración a la lluvia y el sol, a los animales como: monos, osos hormigueros, pumas, venados, murciélagos, serpientes, aves, y a las estructuras vegetales como: hojas, flores y frutos que expresan la vegetación propia de la región. Además, pintaban actividades de subsistencia como la agricultura, caza, pesca, curaciones, entre otras (Porras, 1987)
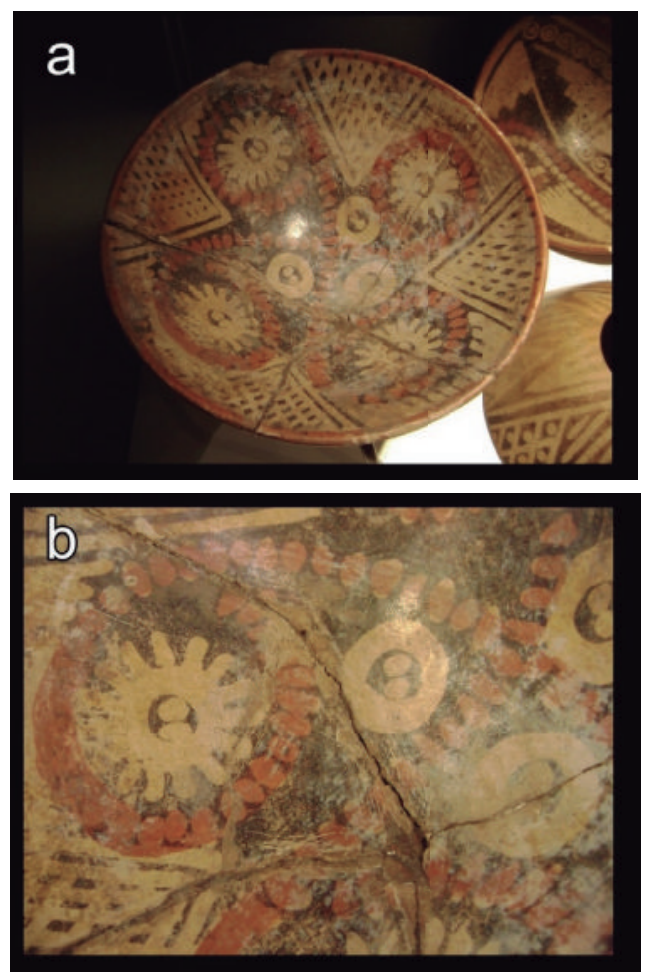


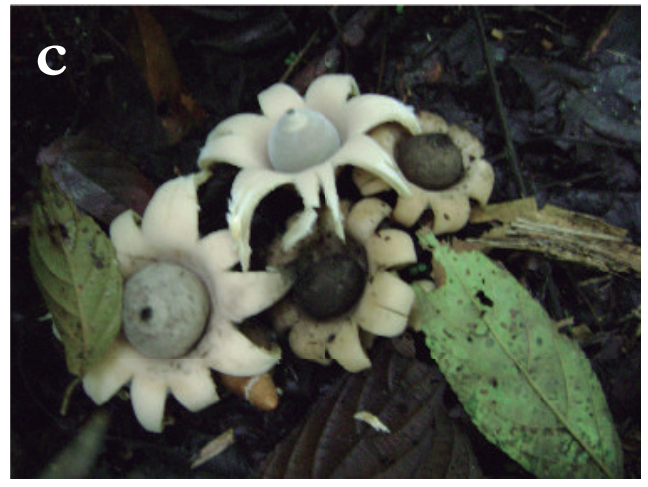

Figura 1. a. Compotera de pie corto perteneciente a la cultura Cuasmal, plato hemisférico con diseños micomórficos con representaciones semejantes al macromycetos agarical Geastrum sp. (c); b. Detalles de lo que parecería tres basidiomas cerrados ubicados en el centro del plato y 4 basidiomas abiertos ubicados en los extremos; se observa estructuras que se asemejan al endoperidio y las lacinias (d). (Foto J. P. Gamboa-Trujillo, Museo Arqueológico Antonio Santiana, Universidad Central del Ecuador. enero de 2009; 2010).

\section{Cultura Manteña}

En lo que concierne a la Cultura Manteña, ubicada en el pasado en las provincias Guayas y Manabí, esta se extendió a lo largo de la Costa Ecuatoriana, desde un poco más al norte de Bahía de Caráquez hasta la Isla de la Puná, tierra adentro hasta el Cerro de Hojas y colinas vecinas del sur de la provincia de Manabí. Se pudo identificar lo que consideraríamos una compotera de pie alargado de $20 \mathrm{~cm}$, cóncava hacia el centro, con borde surcado (apendiculado), de color negro, característica por la presencia de oxigeno durante su cocimiento, técnica usada por los manteños (Porras, 1987) (Figura 2). Esta estructura es posiblemente mi- comórfica y data de $800-1530$ dC. Sus características pueden representar la forma de un macrohongo perteneciente al orden Agaricales, por la presencia de ciertas macroestructuras como el margen apendiculado y algo parecido a una volva en la base. Una forma interesante se aprecia en la vista superior central del píleo en donde se observa una cavidad que servía posiblemente para depositar sustancias 0 bebidas alucinógenas en los eventos ritualísticos (Brau, 1968). La pieza fue estudiada por G.H.S. Bushnell (1950) y depositada en la colección del Museo Arqueológico Antonio Santiana (Porras, 1987).

Por efectos de flujo de información que mantenían los pueblos mesoamericanos con los del hemisferio sur, las actividades de intercambio de productos y de conocimientos locales pudo dar origen a la práctica de rituales similares, utilizando en sus costumbres y creencias organismos morfológicamente parecidos como, en este caso, especies de macromicetos.

Los Manteños eran personas de mucha actividad comercial, además de ser grandes navegantes que surcaron el océano Pacífico hacia Centroamérica (Mesoamérica) y Chile. Las principales mercancías de comercio eran la concha Spondylus sp., tejidos de algodón, principalmente las mantas, por lo que los españoles dieron el nombre de "Manteños". Se ha rescatado de esta cultura cántaros antropomorfos y zoomorfos, figurinas con representaciones ornitológicas, entre otras (Estrada, 1962).

Por esta razón, la pieza descrita es considerada como una herramienta importante dentro de eventos ritualísticos por su forma de cáliz ceremonial, teniendo el mismo uso y apreciación dentro de estas comunidades ancestralmente vinculadas, aunque fabricado en cerámica, al contrario del uso de piedras en Me- 

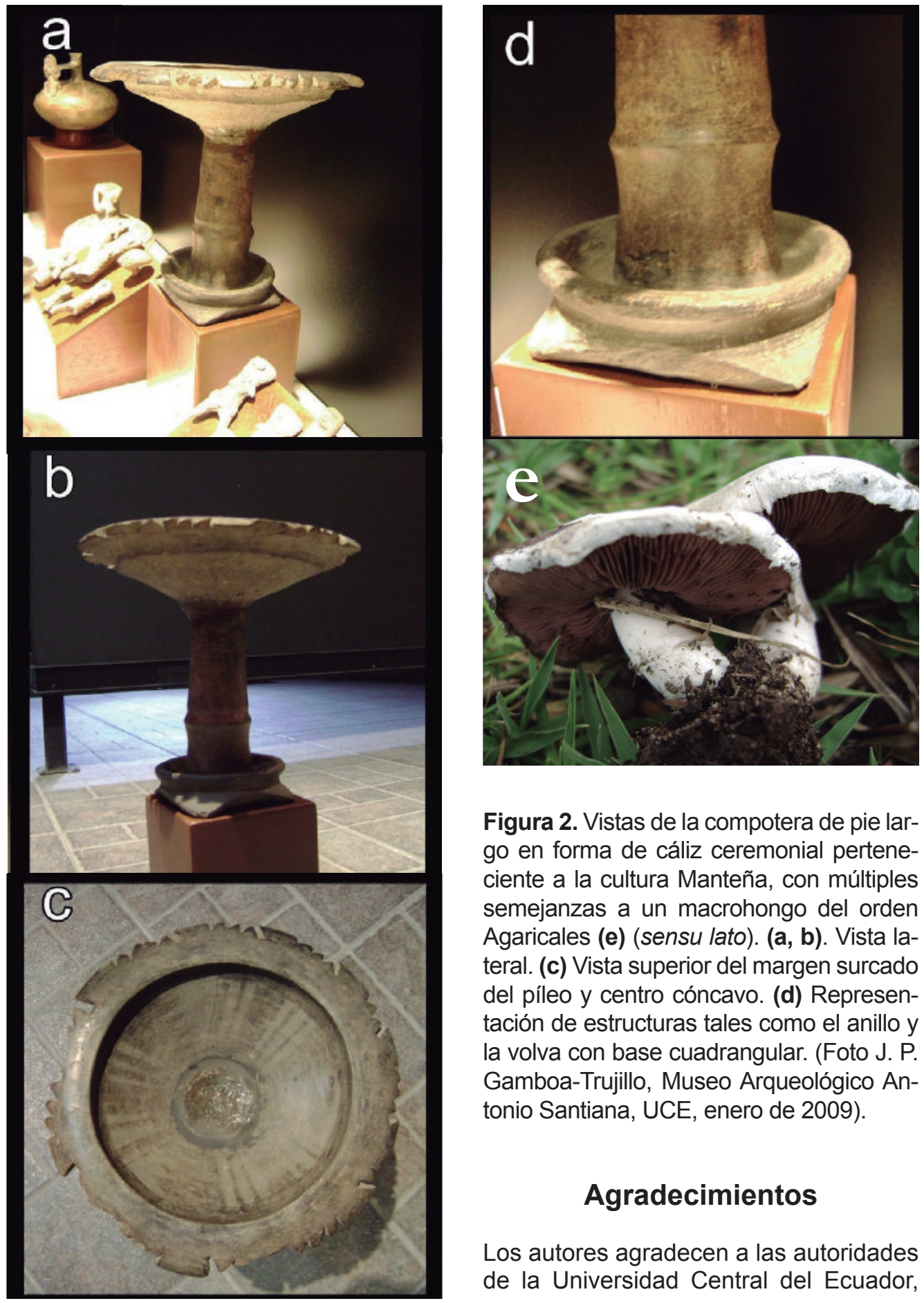

Figura 2. Vistas de la compotera de pie largo en forma de cáliz ceremonial perteneciente a la cultura Manteña, con múltiples semejanzas a un macrohongo del orden Agaricales (e) (sensu lato). (a, b). Vista lateral. (c) Vista superior del margen surcado del píleo y centro cóncavo. (d) Representación de estructuras tales como el anillo y la volva con base cuadrangular. (Foto J. P. Gamboa-Trujillo, Museo Arqueológico Antonio Santiana, UCE, enero de 2009).

\section{Agradecimientos}

Los autores agradecen a las autoridades de la Universidad Central del Ecuador, 
a la comunidad de Agua Blanca, Pto. López; Alejandro y Mentor Gamboa por la ayuda en el manejo de las piezas arqueológicas; in memoriam Dr. Plutarco Naranjo y Dr. Gastón Guzmán por sus sabios y oportunos consejos en la revisión del presente artículo; Dr. Carlos Cerón y Carmita Reyes por el aprendizaje y apoyo (Herbario QAP-UCE), Blgo. José Luis Román Carrión (Museo de Historia Natural Gustavo Orces, Escuela Politécnica Nacional); Leonor Costa Maia, Maria Auxiliadora de Queiroz Cavalcanti, Elaine Malosso, Laise de Holanda Cavalcanti, Ricardo Drechsler-Santos y Felipe Warchow (Universidade Federal de Pernambuco, Brasil). Agradecemos también a Secretaria Nacional de Ciencia y Tecnología de Ecuador (SENESCYT) y a Coordenação de Aperfeiçoamento de Pessoal de Nivel Superior (CAPES, Brasil) por el apoyo financiero.

\section{Bibliografía Citada}

Brau, J. L. 1968. Historie de la Drogue. Editorial Bruguera, Barcelona.

Estrada, E. 1962 Arqueología de Manabí central. Publicaciones del Museo Victor E. Estrada. Vol.7. Guayaquil.

Gamboa-Trujillo, P.,M.De Aro, Flores, S.Gómez y Sanchez M. 2005. Notas Etnomicológicas de los Colonos de la Cuenca del Río Intag, Imbabura-Ecuador. XXIX Jornadas Ecuatorianas de Biología. Manta noviembre 24-26, p.156.

Guzmán,G.2001.Hallucinogenic, medicinal, and Edible Mushrooms in Mexico and Guatemala:Tradition,Myths, and knowledge.International Journal of Medicinal Mushrooms,3:399-408.
Guzmán, G. 2009. The Allucinogenic Mushroms: Diversity, Tradition, Use and Abuse with Special Reference to the Genus Psilocybe en Fungi from Different Enviroments,Misra J.K. \& S.K. Deshmukhleds (eds.) Science Publishers. United States of America.

Grijalba, C.E. 1937. La expedición de Max Uhle a Cuasmal, Protohistoria de Imbabura y Carchi. Editorial Chimborazo. Quito.

Patouillard, N.and de Lagerheim G.(1891).Champignons de L'Equateur, in Collected Mycological. Papers Volumen II, Librarian Rijksherbarium, Leiden.

Porras, P., 1987 Manual de Arqueología, Centro de Investigaciones Arqueológicas, Impreseñal, Quito.

De Sahagún, B., 1569-1582. Historia de las cosas de la Nueva España. edición 1955, Mexíco, D.F.

Schultes y Bright, 1985 Antiguos pectorales de oro: representaciones de Hongos, Boletín Cultural y Bibliográfico, 22(5):pp.3-16.

Schultes, R. E. and Hofmann (1979). Plants of the Gods: Origins of hallucinogenic Use. MacGraw-Hill, New York, USA.

Velandia, C., L. Galindo, K. Mateus, 2008 Micolatria en la iconografia de América del Sur.International Journal of South American Archaeology 3:1-8.

Wasson, R. G. (1980). The Wondrous Mushroom: Mycolatry in Mesoamerica, McGraw-Hill, New York. pp. 209. 